\title{
Proximal femoral nail antirotation and third-generation Gamma nail: which is a better device for the treatment of intertrochanteric fractures?
}

\author{
Yue-Hua Yang ${ }^{1}$, MD, Yu-Ren Wang ${ }^{1}$, MD, Sheng-Dan Jiang ${ }^{1}$, MD, PhD, Lei-Sheng Jiang ${ }^{1}$, MD, PhD
}

\begin{abstract}
INTRODUCTION Proximal femoral nail antirotation (PFNA) and third-generation Gamma nail (Gamma 3) are widely used in the treatment of intertrochanteric fractures. However, it remains unclear which device achieves better clinical and radiographic outcomes when treating intertrochanteric fractures.

METHODS This study comprised 239 patients with intertrochanteric fractures treated with either PFNA or Gamma 3 for a minimum of 12 months. During surgery, the operative time, image intensifier time and amount of blood loss were recorded. Following surgery, we assessed reduction quality and implant position. At the final follow-up, postoperative complications, including femoral shaft fracture, cutout, reoperation, pneumonia, urinary tract infection, cerebral infarction, cardiac infarction and decubital ulcer, were recorded. In addition, walking ability was assessed using the Parker-Palmer mobility score.

RESULTS No difference was found in the operative time, image intensifier time and amount of blood loss between patients treated with PFNA and those treated with Gamma 3. The reduction quality of fractures treated with Gamma 3 was better than those treated with PFNA. However, there were no significant differences in implant position, walking ability and postoperative complications between the two groups. Although Gamma 3 resulted in better reduction quality, it did not provide any advantages in walking ability and postoperative complications when compared with PFNA.

CONCLUSION Therefore, we conclude that both PFNA and Gamma 3 are safe and reliable devices for the treatment of intertrochanteric fractures.
\end{abstract}

Keywords: intertrochanteric fractures, intramedullary fixation

\section{INTRODUCTION}

Intertrochanteric fractures constitute one of the most common fractures of the hip, occurring mainly in elderly people with osteoporosis. Due to an ageing population, the incidence of intertrochanteric fractures is expected to continue to increase. The goal of treatment is fracture reduction and stable fixation in order to allow immediate mobilisation, thereby reducing the incidence of complications such as pneumonia, urinary tract infection and cardiovascular events.

For many years, the sliding hip screw and plate fixation technique has been considered the gold standard in the treatment of intertrochanteric fractures. ${ }^{(1)}$ Unfortunately, it has a reported failure rate of $8 \%-13 \% .^{(2,3)}$ Implant failure is associated with revision surgery, prolonged in-hospital stay, increased risk of infections, and a diminished chance of regaining autonomy and mobility. Therefore, some authors have recommended prosthetic replacement to treat intertrochanteric fractures in older patients with severe osteoporosis or comminution. ${ }^{(4,5)}$ However, as the population ages and the activity level among the elderly increases, the durability of the endoprosthesis also decreases more rapidly, warranting replacement of the device with a second surgery, which poses an even higher risk for complications. In a prospective, randomised study comparing the use of long-stem cementless calcar-replacement hemiarthroplasty and proximal femoral nail in elderly patients with unstable intertrochanteric fractures, the latter has been found to provide superior clinical outcomes. ${ }^{(6)}$ There is now increasing interest in the use of intramedullary nailing to treat intertrochanteric fractures, especially unstable fractures. Both proximal femoral nail antirotation (PFNA) and third-generation Gamma nail (Gamma 3) are widely used in the treatment of intertrochanteric fractures. However, it remains unclear which device provides better clinical and radiographic outcomes.

The aim of this study was to investigate the clinical and radiographic outcomes of these two intramedullary devices in the treatment of intertrochanteric fractures, so as to help guide clinical decision-making.

\section{METHODS}

From January 2007 to February 2010, a total of 239 patients with intertrochanteric fractures treated in Xinhua Hospital, Shanghai, using either PFNA ( $n=115)$ or Gamma $3(n=124)$, were enrolled in the study. Inclusion criteria were: (a) age

${ }^{1}$ Department of Orthopaedic Surgery, Xinhua Hospital, Shanghai Jiaotong University School of Medicine, Shanghai, China

Correspondence: Prof Sheng-Dan Jiang, Professor, Department of Orthopaedic Surgery, Xinhua Hospital, Shanghai Jiaotong University School of Medicine, 1665 Kongjiang Road, Shanghai 200092, China. jiangsd@126.com 
over 60 years; and (b) nonpathological intertrochanteric fractures. Patients with pathological intertrochanteric fractures were excluded.

The patients were assigned to undergo either PFNA (Synthes $\mathrm{GmbH}$, Oberdorf, Switzerland) or Gamma 3 (Stryker, Mahwah, NJ, USA) fixation, according to the physician's decision. Closed reduction and internal fixation with intramedullary hip nail was performed in all patients on a standard radiolucent table under image intensifier control. We used a $130^{\circ}$ intramedullary nail (short version) in both systems. The helical blade of PFNA and the lag screw of Gamma 3 were inserted into the lower half of the femoral head on anteroposterior view and in the middle-third of the femoral head on lateral view. After insertion of the helical blade or lag screw, longitudinal traction was released to reduce fracture gap. One distal dynamic interlocking screw was subsequently inserted in all cases. All the operations were performed by two senior attending surgeons from Xinhua Hospital.

All the patients were allowed protected weight bearing with a walking stick for three months. Prophylactic antibiotics were administered, and prophylactic low-molecularweight heparin was given subcutaneously for five weeks postoperatively. Most patients were discharged from the hospital after 5-10 days, either to their previous residences or to temporary rehabilitation hospitals.

All the patients' records, including gender, age, American Society of Anesthesiologists (ASA) class rating and fracture pattern according to Evans classification, were complete. Reduction quality and implant position were recorded after surgery. Postoperative follow-up was undertaken at 1, 3, 6 and 12 months, and yearly thereafter. Anteroposterior and lateral plain radiographs were obtained at each visit.

The quality of fracture reduction was assessed using the measurement of the postoperative fracture gap $(\mathrm{mm})$ and the Garden Alignment Index. ${ }^{(7)}$ The fracture gap, which was measured using the first postoperative anteroposterior and lateral radiographs, was classified as good (0-3 mm); acceptable (3-5 mm); or poor (> $5 \mathrm{~mm}$ ). The repositioning of the head and neck fragment was evaluated according to the Garden Alignment Index. The average physiologic angle of the femur shaft and medial trabecular structures is $160^{\circ}$ and $180^{\circ}$ in the anteroposterior and axial projections, respectively. The results were classified as very good (anteroposterior $160^{\circ}$ ); good (anteroposterior $180^{\circ}-160^{\circ}$ ); acceptable (anteroposterior $160^{\circ}-150^{\circ}$ ); or poor (anteroposterior $<150^{\circ} /$ lateral not $180^{\circ}$ ). ${ }^{(7)}$ The implant position in the femoral head was recorded using Cleveland zones $^{(8)}$ and tip apex distance (TAD). ${ }^{(9)}$ To determine the implant position in the femoral head, the anteroposterior and lateral radiographs were scanned with a matrix of the nine Cleveland zones with the zone number at the centre of the head. TAD was calculated by measuring the distance between the tip of the proximal implant (lag screw) to the apex of the femoral head on
Table I. Preoperative characteristics of the patients.

\begin{tabular}{lccc}
\hline Parameter & \multicolumn{2}{c}{ No. of patients (\%) } & \multirow{2}{*}{ p-value } \\
\cline { 2 - 3 } & $\begin{array}{c}\text { PFNA } \\
\text { (n = 115) }\end{array}$ & $\begin{array}{c}\text { Gamma 3 } \\
\text { (n = 124) }\end{array}$ & \\
\hline Gender & $39(33.9)$ & $38(30.6)$ & $>0.05$ \\
$\quad$ Male & $76(66.0)$ & $86(69.4)$ & \\
Female & $78.9(61-93)$ & $81.6(62-101)$ & $>0.05$ \\
Age* (yrs) & & & $>0.05$ \\
Side of fracture & $63(54.8)$ & $78(62.9)$ & \\
Left & $52(45.2)$ & $46(37.1)$ & $>0.05$ \\
Right & & & \\
Evans classification & $6(5.2)$ & $9(7.3)$ & \\
I & $13(11.3)$ & $12(9.7)$ & \\
II & $64(55.7)$ & $71(57.3)$ & \\
III & $25(21.7)$ & $21(16.9)$ & \\
IV & $7(6.1)$ & $11(8.9)$ & \\
V & & & \\
ASA score & $45(39.1)$ & $54(43.5)$ & \\
II & $56(48.7)$ & $59(47.6)$ & \\
III & $14(12.2)$ & $11(8.9)$ & \\
IV & & & \\
\hline IV & & & \\
\hline
\end{tabular}

*Data is presented as median (range).

ASA: American Society of Anesthesiologists; PFNA: proximal femoral nail antirotation

the anteroposterior and lateral radiographs after applying Baumgartens formula.

Postoperative complications, including femoral shaft fracture, cutout, reoperation, pneumonia, urinary tract infection, cerebral infarction, cardiac infarction and decubital ulcer, were recorded. Walking ability was assessed using the Parker-Palmer ${ }^{(10)}$ mobility score (0-9 points) at the final follow-up.

For both groups, data were represented as mean and standard deviation (SD) for continuous response variables, or numbers and percentages for discrete variables. For discrete variables, chi-square test was used to compare the differences between the two groups, whereas for continuous variables, Student's $t$-test was used. Before analysis, the $\mathrm{p}$-value was set at 0.05 for each test.

\section{RESULTS}

The preoperative characteristics of patients who were treated with either PFNA or Gamma 3 are shown in Table I. No significant differences were found between the two groups in terms of gender, age, side of fracture, type of fracture and ASA score. At the final follow-up, 5 patients died and 12 were lost to follow-up in the PFNA group, while in the Gamma 3 group, 4 died and 17 were lost to follow-up. The mean follow-up time was 29 (range 12-63) months.

The mean time between trauma and operation was 2.4 days (PFNA: 2.6 days; Gamma 3: 2.2 days; overall: min 1 day, $\max 5$ days). No significant difference was found in the operative time, image intensifier time and quantity of blood loss between the two groups (Table II). No intraoperative complication was observed in any of the patients.

Fracture reduction was assessed using the measurement of the fracture gap and the Garden Alignment Index. The mean 
Table II. Comparison of operative time, image intensifier time and amount of blood loss between the two groups.

\begin{tabular}{llcl}
\hline Parameter & \multicolumn{2}{c}{ Mean \pm SD } & p-value \\
\cline { 2 - 3 } & PFNA & Gamma 3 & \\
\hline Operative time (min) & $22 \pm 7$ & $23 \pm 9$ & $>0.05$ \\
Image intensifier time (s) & $18 \pm 4$ & $20 \pm 3$ & $>0.05$ \\
Blood loss $(\mathrm{mL})$ & $73 \pm 22$ & $81 \pm 34$ & $>0.05$ \\
\hline
\end{tabular}

Gamma 3: third-generation Gamma nail; PFNA: proximal femoral nail antirotation; SD: standard deviation

Table III. Comparison of postoperative fracture gap measurements between the two groups.

\begin{tabular}{lccc}
\hline Parameter & \multicolumn{2}{c}{ No. of patients (\%) } & \multirow{2}{*}{ p-value } \\
\cline { 2 - 3 } & $\begin{array}{c}\text { PFNA } \\
(\mathbf{n}=\mathbf{1 1 5})\end{array}$ & $\begin{array}{c}\text { Gamma 3 } \\
(\mathbf{n}=\mathbf{1 2 4})\end{array}$ & \\
\hline Fracture gap* (mm) & $3.6 \pm 0.7$ & $1.9 \pm 0.4$ & $<0.05$ \\
$<3$ & $51(44.3)$ & $102(82.3)$ & \\
$3-5$ & $57(49.6)$ & $22(17.7)$ & \\
$>5$ & $7(6.1)$ & 0 & $<0.05$ \\
\hline
\end{tabular}

*Data is presented as mean \pm standard deviation.

Gamma 3: third-generation Gamma nail; PFNA: proximal femoral nail antirotation

fracture gap in the PFNA group was significantly greater than that in the Gamma 3 group. In the PFNA group, the fracture gap measurement was $<3 \mathrm{~mm}$ in 51 cases, $<5 \mathrm{~mm}$ in 57 cases and $>5 \mathrm{~mm}$ in 7 cases. In the Gamma 3 group, the measurement was $<3 \mathrm{~mm}$ in 102 cases and $<5 \mathrm{~mm}$ in 22 cases, with no case having a measurement $>5 \mathrm{~mm}$ (Table III). Statistical analysis between the two groups revealed a significant difference in the reduction quality, in favour of patients treated with Gamma 3.

Using the Garden Alignment Index, the reduction quality of fractures that were treated with PFNA was graded as very good in 27 cases; good in 55; acceptable in 24; and poor in 9. In fractures treated with Gamma 3, 32 cases were documented as very good; 57 as good; 25 as acceptable; and 10 as poor. The mean Garden Alignment Index of the PFNA group was similar to that of the Gamma 3 group, and statistical analysis between the two groups revealed no significant difference (Table IV).

The implant position was recorded according to the Cleveland zones and TAD. The tip of the helical blade of PFNA was within Cleveland zone 5 in 91 (79.1\%) cases; zone 6 in $13(11.3 \%)$ cases; zone 4 in $3(2.6 \%)$ cases; and zone 8 in $2(1.7 \%)$ cases. The tip of the lag screw of Gamma 3 was within the Cleveland zone 5 in 97 (78.2\%) cases; zone 6 in $22(17.7 \%)$ cases; zone 4 in $3(2.4 \%)$ cases; and zone 8 in $2(1.6 \%)$ cases. Statistical analysis between the two groups revealed no significant difference. On average, the TAD for the PFNA group was $16.7 \mathrm{~mm}$ for the spiral blade, while that of the Gamma 3 group was $18.6 \mathrm{~mm}$ for the lag screw. Regardless of whether patients received PFNA or Gamma 3 fixation, there was no significant change in TAD immediately after surgery and at one month after surgery. Although, TAD decreased significantly from the time immediately
Table IV. Comparison of postoperative Garden Alignment Index between the two groups.

\begin{tabular}{|c|c|c|c|}
\hline \multirow[t]{2}{*}{ Parameter } & \multicolumn{2}{|c|}{ No. of patients (\%) } & \multirow[t]{2}{*}{ p-value } \\
\hline & $\begin{array}{c}\text { PFNA } \\
(n=115)\end{array}$ & $\begin{array}{l}\text { Gamma } 3 \\
(n=124)\end{array}$ & \\
\hline Garden Alignment & $163 \pm 24$ & $165 \pm 19$ & $>0.05$ \\
\hline \multicolumn{4}{|l|}{ Index ${ }^{*}\left({ }^{\circ}\right)$} \\
\hline 160 & $27(23.5)$ & $32(25.8)$ & \\
\hline $180-160$ & $55(47.8)$ & $57(46.0)$ & \\
\hline $160-150$ & $24(20.9)$ & $25(20.2)$ & \\
\hline$<150$ & $9(7.8)$ & $10(8.1)$ & $>0.05$ \\
\hline
\end{tabular}

* Data is presented as mean \pm standard deviation.

Gamma 3: third-generation Gamma nail; PFNA: proximal femoral nail antirotation

after surgery to the third month after surgery, no significant decrease was observed at the twelfth month after surgery when compared to the TAD at the third month (Table V).

Complications that occurred during the postoperative period are summarised in Table VI. There were two cases of cutout with TAD $<20 \mathrm{~mm}$ (TAD of $18 \mathrm{~mm}$ and $19 \mathrm{~mm}$, respectively). Of these two cases, one was treated with PFNA, resulting in good screw position but poor fracture reduction, while the other was treated with Gamma 3 with resultant good screw position and fracture reduction but severe osteoporosis. There was no statistical difference in the incidence of lag screw cutout between patients with TAD values $>25 \mathrm{~mm}$ and those with TAD values $<25 \mathrm{~mm}$. Due to the occurrence of cutout, total hip arthroplasty was performed in one patient, but surgery was declined by the other patient. No femoral shaft fracture was found in any of the patients within the follow-up period. There was no significant difference in the frequency of cutout, reoperation, pneumonia, urinary tract infection, cerebral infarction, cardiac infarction and decubital ulcer between the two groups.

At the final follow-up, $73(74.5 \%)$ of the remaining 98 patients in the PFNA group (5 died and 12 were lost to follow-up) achieved independent walking, 21 (21.4\%) required a walking aid and $4(4.1 \%)$ were not ambulatory. The corresponding values in the Gamma 3 group $(n=103 ; 4$ died and 17 were lost to follow-up) were $80(77.7 \%), 18(17.5 \%)$ and $5(4.9 \%)$, respectively ( $p>0.05)$. The mean preoperative Parker-Palmer mobility score was 7.9 for the PFNA group and 8.1 for the Gamma 3 group. The mean postoperative ParkerPalmer mobility score was 7.1 for the PFNA group and 7.0 for the Gamma 3 group. Statistical analysis of the two treatment groups revealed no significant difference between them.

\section{DISCUSSION}

In our study, the patients in both the PFNA and Gamma 3 groups had good outcomes, with few complications and a failure rate of $0.8 \%$. Both PFNA and Gamma 3 have the advantages of high union rate, early postoperative mobilisation, short image intensifier and operative times, less blood loss and fewer postoperative complications. We also found PFNA and Gamma 3 osteosynthesis to be the methods 
Table V. TAD immediately after surgery and at 1, 3, 6 and 12 months after surgery.

\begin{tabular}{lccccc}
\hline Parameter & \multicolumn{5}{c}{ Mean TAD \pm SD } \\
\cline { 2 - 6 } & Postop & $\mathbf{1}$ mth postop & 3 mths postop & $\mathbf{6}$ mths postop & $\mathbf{1 2}$ mths postop \\
\hline PFNA & $16.7 \pm 2.4$ & $15.6 \pm 2.2$ & $13.3 \pm 1.7$ & $12.9 \pm 1.6$ & $12.5 \pm 1.5$ \\
Gamma 3 & $18.6 \pm 2.7$ & $18.1 \pm 2.3$ & $14.2 \pm 1.9$ & $13.7 \pm 1.5$ & $13.6 \pm 1.7$ \\
\hline
\end{tabular}

Gamma 3: third-generation Gamma nail; PFNA: proximal femoral nail antirotation; SD: standard deviation; TAD: tip apex distance

Table VI. Comparison of postoperative complications at the final follow-up.

\begin{tabular}{lrcc}
\hline Complication & \multicolumn{2}{c}{ No. of patients (\%) } & p-value \\
\cline { 2 - 3 } & $\begin{array}{c}\text { PFNA } \\
(\mathbf{n}=\mathbf{1 0 3})\end{array}$ & $\begin{array}{c}\text { Gamma 3 } \\
(\mathbf{n}=\mathbf{1 0 7})\end{array}$ & \\
\hline Cutout & $1(1.0)$ & $1(0.9)$ & $>0.05$ \\
Reoperation & $1(1.0)$ & $1(0.9)$ & $>0.05$ \\
Pneumonia & $9(8.7)$ & $7(6.5)$ & $>0.05$ \\
Urinary tract infection & $5(4.9)$ & $6(5.6)$ & $>0.05$ \\
Cerebral infarction & $5(4.9)$ & $7(6.5)$ & $>0.05$ \\
Cardiac infarction & $2(1.9)$ & $1(0.9)$ & $>0.05$ \\
Decubital ulcer & $3(2.9)$ & $2(1.9)$ & $>0.05$ \\
Death & $5(4.9)$ & $4(3.7)$ & $>0.05$ \\
\hline
\end{tabular}

Note: 12 patients were lost to follow-up in the PFNA group, while 17 were lost to follow-up in the Gamma 3 group.

Gamma 3: third-generation Gamma nail; PFNA: proximal femoral nail antirotation

of choice for the surgical treatment of intertrochanteric fractures in the elderly.

Our study showed no significant differences in image intensifier time, operative time, amount of blood loss and postoperative complications between patients treated with PFNA and those treated with Gamma 3. However, $\mathrm{Xu}$ et $\mathrm{al}^{(11)}$ reported that PFNA fixation for the treatment of intertrochanteric fractures resulted in less blood loss and shorter fluoroscopy time as compared with Gamma 3. These contradictory findings could be attributed to differences in the level of experience of the surgeons. We found both PFNA and Gamma 3 to be reliable implants for treating intertrochanteric fractures.

There were two cases of cutouts in our study. One had been treated with PFNA, while the other with Gamma 3, suggesting that there is no significant difference in the frequency of cutouts between patients treated with the two devices. In a small cohort of patients treated with a cephalomedullary implant, Baumgaertner et $\mathrm{al}^{(9)}$ documented that the ideal position of the lag screw was in the centre-centre position. The right position of the lag screw and helical blade near the centre of the femoral head and neck, in both the anteroposterior and lateral views, is critical. TAD, which represents both the position and depth of a screw in the femoral neck and head, was shown to be the most important predictive factor for the occurrence of a cutout. ${ }^{(12,13)}$ Geller et al reported a high incidence (44\%) of cutouts in intertrochanteric fractures that were surgically fixed with a TAD of $>25 \mathrm{~mm} .{ }^{(14)}$ However, no fixation failure was observed in our series, possibly due to the small number of intertrochanteric fractures that were surgically fixed with a TAD of $>25 \mathrm{~mm}$. No cutout was seen in patients with a TAD of $<25 \mathrm{~mm}$ in Geller et al's study. ${ }^{(14)}$ In another study involving 1,150 patients with intertrochanteric fractures treated with $135^{\circ}$ dynamic hip screw, Hsueh et al reported 11 cutouts among cases with a TAD of $<25 \mathrm{~mm}, 2$ cutouts among cases with a TAD of $<20 \mathrm{~mm}$, and no screw cutout in cases with a TAD of $<15 \mathrm{~mm} .{ }^{(15)}$ These findings are similar to those of our study, where two cutouts were noted in cases with a TAD of $<20 \mathrm{~mm}$, and no screw cutout in cases with a TAD of $<15 \mathrm{~mm}$. In view of this, we suggest that TAD should be kept below $15 \mathrm{~mm}$ so as to avoid cutouts of the lag screw or helical blade. Although TAD is the most important factor in predicting lag screw cutouts, it is not the only factor, as fracture pattern, fracture reduction, bone quality and age are also important predictive factors. In addition, it is interesting that in our study, no significant change was observed in TAD immediately after surgery and at one month after surgery, regardless of the type of treatment (PFNA or Gamma 3), whereas TAD decreased significantly from the time immediately after surgery to the third month after surgery. This could be due to blockage of the gliding mechanism, which causes the helical blade and lag screw to protrude from the headneck fragment during weight bearing.

According to the Cleveland zones, there was no significant difference in implant position between the two groups. Due to the physiological $12^{\circ}$ anteversion of the neck of the femur, Cleveland zones 5, 6, 8 and 9 are in an area of no rotational force. We placed most of the helical blade tips or lag screw tips in zone 5 (78.6\%). Implant tips placed in zones 4 or 7 are, from the biomechanical point of view, in the area of rotational forces. This might account for the rotation of the head and neck fragment, resulting in cutout. However, no cutout was observed in our patients with helical blades or lag screws placed in zone 4 .

No intraoperative femoral fracture was observed in our study. However, Yaozeng et al reported that femoral shaft fractures were observed in 6 of the 107 patients with intertrochanteric fractures in their study. ${ }^{(11)}$ The relatively shorter proximal femoral length and femoral medullary cavity diameter in the Chinese population as compared to the Europeans and Americans may explain this phenomenon. ${ }^{(16)}$ Femoral shaft fractures may also result from insufficient reaming of the intramedullary cavity. Thus, ensuring sufficient reaming of the intramedullary cavity can decrease the incidence of intraoperative femoral shaft fractures. 
Our study demonstrated that the reduction quality of fractures treated with Gamma 3 was better than that treated with PFNA. Two factors may explain this phenomenon. One is that Gamma nail can provide better compression of bone fragments than PFNA. The other is that the helical blade of PFNA, which is inserted into the femoral neck without drilling, may push the head and neck fragment medially, leading to distraction of the bone fragments.

The ambulatory status of patients after an operation for an intertrochanteric fracture depends on different factors. ${ }^{(17-19)}$ The overall walking competence was similar in patients treated with PFNA and those treated with Gamma 3. This is likely because both PFNA and Gamma 3 allow for accurate nail placement, leading to secure and stable fixation with good clinical outcomes.

In conclusion, although treatment with Gamma 3 results in better reduction quality than PFNA, the clinical outcome of Gamma 3 is comparable to that of PFNA. Both PFNA and Gamma 3 are good intramedullary osteosynthesis for intertrochanteric fractures due to their low complication rates and good clinical results. Our conclusions, however, should be confirmed in further randomised controlled studies, as our study was undertaken prospectively, and thus may be limited due to the nature of its design.

\section{REFERENCES}

1. Wolfgang GL, Bryant MH, O'Neill JP. Treatment of intertrochanteric fracture of the femur using sliding screw plate fixation. Clin Orthop Relat Res 1982; 163:148-58.

2. Bannister GC, Gibson AG, Ackroyd CE, Newman JH. The fixation and prognosis of trochanteric fractures. A randomized prospective controlled trial. Clin Orthop Relat Res 1990; 254:242-6.

3. Simpson AH, Varty K, Dodd CA. Sliding hip screws: modes of failure. Injury 1989; 20:227-31.

4. Haentjens P, Lamraski G. Endoprosthetic replacement of unstable, comminuted intertrochanteric fracture of the femur in the elderly, osteoporotic patient: a review. Disabil Rehabil 2005; 27:1167-80.
5. Stappaerts KH, Deldycke J, Broos PL, et al. Treatment of unstable peritrochanteric fractures in elderly patients with a compression hip screw or with the Vandeputte (VDP) endoprosthesis: a prospective randomized study. J Orthop Trauma 1995; 9:292-7.

6. Kim SY, Kim YG, Hwang JK. Cementless calcar-replacement hemiarthroplasty compared with intramedullary fixation of unstable intertrochanteric fractures. A prospective, randomized study. J Bone Joint Surg Am 2005; 87:2186-92.

7. Lenich A, Mayr E, Rüter A, Möckl Ch, Füchtmeier B. First results with the trochanter fixation nail (TFN): a report on 120 cases. Arch Orthop Trauma Surg 2006; 126:706-12.

8. Cleveland M, Bosworth DM, Thompson FR, Wilson HJ Jr, Ishizuka T. A ten-year analysis of intertrochanteric fractures of the femur. J Bone Joint Surg Am 1959; 41-A:1399-408.

9. Baumgaertner MR, Curtin SL, Lindskog DM, Keggi JM. The value of the tipapex distance in predicting failure of fixation of peritrochanteric fractures of the hip. J Bone Joint Surg Am 1995; 77:1058-64.

10. Parker MJ, Palmer CR. A new mobility score for predicting mortality after hip fracture. J Bone Joint Surg Br 1993; 75:797-8.

11. Yaozeng X, Dechun G, Huilin Y, Guangming Z, Xianbin W. Comparative study of trochanteric fracture treated with the proximal femoral nail antirotation and the third generation of gamma nail. Injury 2010; 41:1234-8.

12. Rubio-Avila J, Madden K, Simunovic N, Bhandari M. Tip to apex distance in femoral intertrochanteric fractures: a systematic review. J Orthop Sci $2013 ; 18: 592-8$.

13. Andruszkow $H$, Frink M, Frömke C, et al. Tip apex distance, hip screw placement, and neck shaft angle as potential risk factors for cut-out failure of hipscrews after surgical treatment of intertrochanteric fractures. Int Orthop 2012; 36:2347-54.

14. Geller JA, Saifi C, Morrison TA, Macaulay W. Tip-apex distance of intramedullary devices as a predictor of cut-out failure in the treatment of peritrochanteric elderly hip fractures. Int Orthop 2010; 34:719-22.

15. Hsueh KK, Fang CK, Chen CM, et al. Risk factors in cutout of sliding hip screw in intertrochanteric fractures: an evaluation of 937 patients. Int Orthop 2012; 34:1273-6.

16. Leung KS, Procter P, Robioneck B, Behrens K. Geometric mismatch of the Gamma nail to the Chinese femur. Clin Orthop Relat Res 1996; (323):42-8.

17. Koval KJ, Sala DA, Kummer FJ, Zuckerman JD. Postoperative weightbearing after a fracture of the femoral neck or an intertrochanteric fracture. J Bone Joint Surg Am 1998; 80:352-6.

18. Koval KJ, Skovron ML, Aharonoff GB, Meadows SE, Zuckerman JD. Ambulatory ability after hip fracture. A prospective study in geriatric patients. Clin Orthop Relat Res 1995; (310):150-9.

19. van der Sluijs JA, Walenkamp GH. How predictable is rehabilitation after hip fracture? A prospective study of 134 patients. Acta Orthop Scand 1991; 62:567-72. 